\title{
A New Radiative Transfer Method for Solar Radiation in a Vertically Internally Inhomogeneous Medium
}

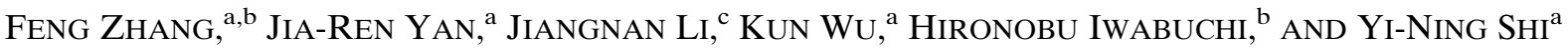 \\ ${ }^{a}$ Key Laboratory of Meteorological Disaster, Ministry of Education, Nanjing University of Information \\ Science and Technology, Nanjing, China \\ ${ }^{\mathrm{b}}$ Center for Atmospheric and Oceanic Studies, Graduate School of Science, Tohoku University, Sendai, Japan \\ ${ }^{\mathrm{c}}$ Canadian Centre for Climate Modelling and Analysis, Environment and Climate Change Canada, University of Victoria, \\ Victoria, British Columbia, Canada
}

(Manuscript received 5 April 2017, in final form 28 September 2017)

\begin{abstract}
The problem of solar spectral radiation is considered in a layer-based model, with scattering and absorption parallel to the plane for each medium (cloud, ocean, or aerosol layer) and optical properties assumed to be vertically inhomogeneous. A new radiative transfer (RT) method is proposed to deal with the variation of vertically inhomogeneous optical properties in the layers of a model for solar spectral radiation. This method uses the standard perturbation method to include the vertically inhomogeneous RT effects of cloud and snow. The accuracy of the new inhomogeneous RT solution is investigated systematically for both an idealized medium and realistic media of cloud and snow. For the idealized medium, the relative errors in reflection and absorption calculated by applying the homogeneous solution increase with optical depth and can exceed $20 \%$. However, the relative errors when applying the inhomogeneous RT solution are limited to $4 \%$ in most cases. Observations show that stratocumulus clouds are vertically inhomogeneous. In the spectral band of $0.25-0.69 \mu \mathrm{m}$, the relative error in absorption with the inhomogeneous solution is $1.4 \%$ at most, but that with the homogeneous solution can be up to $7.4 \%$. The effective radius of snow varies vertically. In the spectral band of $0.25-0.69 \mu \mathrm{m}$, the relative error in absorption with the homogeneous solution can be as much as $72 \%$ but is reduced to less than $40 \%$ by using the inhomogeneous solution. At the spectral wavelength of $0.94 \mu \mathrm{m}$, the results for reflection and absorption with the inhomogeneous solution are also more accurate than those with the homogeneous solution.
\end{abstract}

\section{Introduction}

Radiative transfer (RT) is a key issue in climate modeling and remote sensing. In most numerical radiative transfer algorithms, the atmosphere is divided into many homogeneous layers. The inherent optical properties (IOPs) are then fixed within each layer, and variations of IOPs inside each layer are ignored, effectively regarding each layer as internally homogeneous. The standard RT solutions are based on this assumption of internal homogeneity (Lenoble 1985; Toon et al. 1989; Fu et al. 1997; Li et al. 2005; Zhang and Li 2013), which cannot resolve within-layer vertical inhomogeneity.

It has been well established by observation that cumulus and stratocumulus clouds (hereinafter collectively referred to as cumulus clouds) are inhomogeneous, both horizontally and vertically (Vane et al. 2006; Boutle et al. 2014; Young et al. 2013; Luo et al. 2014; Cheng et al. 2015).

Corresponding author: Feng Zhang, feng_zhang126@126.com
Inside a cumulus cloud, the liquid water content (LWC) and the cloud droplet size distribution vary with height, so the IOPs of cloud droplets depend on vertical height. For snow, the mean grain size of snow packs generally increases with time (Nakaya 1954; LaChapelle 1969; Flanner and Zender 2006). A wide variety of research (Arai 1965; Colbeck 1975; Gow 1969; Hobbs 1974; Mellor 1977; Sommerfeld and LaChapelle 1970) suggests that the average grain radius is in the range of $20-100 \mu \mathrm{m}$ for new snow, 100-300 $\mu \mathrm{m}$ for fine-grain older snow, and $1.0-1.5 \mathrm{~mm}$ for old snow near the melting point. Therefore, snow grain size varies with snow depth, and the IOPs of snow are vertically inhomogeneous. In addition, the aerosol concentrations in the atmosphere are vertically inhomogeneous ( $\mathrm{Li}$ et al. 2008; von Salzen et al. 2013; Guo et al. 2016; Qin et al. 2016, 2017; Wang et al. 2015).

How to deal with vertical internal inhomogeneity in RT models is an interesting topic for researchers. Li et al. (1994) developed a Monte Carlo cloud model that can be used to investigate photon transport in 
inhomogeneous clouds by considering internal variation of the optical properties. Their model showed that when overcast clouds become broken clouds, the difference in reflectance at large solar zenith angles between vertically inhomogeneous clouds and their plane-parallel counterparts can be as much as $10 \%$. However, the Monte Carlo method is time consuming and not applicable to climate models or remote sensing (Liou 2002). The albedo of inhomogeneous mixed-phase clouds at visible wavelengths could be obtained by using a Monte Carlo method to compare such clouds with planeparallel homogeneous clouds (Macke et al. 1998).

In principle, the vertical-inhomogeneity problem of the $\mathrm{RT}$ process can be solved by increasing the number of layers of the climate model. However, it is time consuming to increase the vertical resolution of a climate model. Typically, there are only $30-100$ layers in a climate model (von Salzen et al. 2013), which is not high enough to resolve the cloud vertical inhomogeneity. For snowcovered ground, most climate models consider only the change in surface albedo due to the snow. Recently, the RT process has been extended to snow by treating it as either a single layer or a double layer (Namazi et al. 2015), even though the parameterization of snow-layer IOPs was proposed a long time ago (Wiscombe and Warren 1980; Warren and Wiscombe 1980). To completely address the problem of vertical inhomogeneity with only a limited number of layers in a climate model, the standard RT method must be extended to deal with the vertical inhomogeneity internal to each model layer. The main purpose of this study is to develop a new inhomogeneous RT solution. This solution follows a perturbation method: the zeroth-order solution is the Eddington approximation for homogeneous layers, with a first-order perturbation to account for the inhomogeneity effect. In section 2 , the basic theory of radiative transfer is introduced, and the new inhomogeneous RT solution is presented. In section 3, the accuracy of the new solution is examined for a wide range of idealized cases. Furthermore, this inhomogeneous RT solution is applied to cloud and snow as realistic examples to demonstrate the practicality of this new method. A summary is given in section 4 .

\section{RT solution for an inhomogeneous layer}

The azimuthally averaged solar radiative transfer equation is

$$
\begin{aligned}
\mu \frac{d I(\tau, \mu)}{d \tau}= & I(\tau, \mu)-\frac{\omega(\tau)}{2} \int_{-1}^{1} I\left(\tau, \mu^{\prime}\right) P\left(\tau, \mu, \mu^{\prime}\right) d \mu^{\prime} \\
& -\frac{\omega(\tau)}{4 \pi} F_{s} e^{-\tau / \mu_{0}} P\left(\tau, \mu,-\mu_{0}\right),
\end{aligned}
$$

where $\mu$ is the cosine of the zenith angle ( $\mu>0$ and $\mu<0$ refer to upward and downward radiation, respectively), $P\left(\tau, \mu, \mu^{\prime}\right)$ is the scattering phase function, $\tau$ is the optical depth ( $\tau=0$ and $\tau=\tau_{0}$ refer to the top and bottom of the medium, respectively), $\omega(\tau)$ is the single-scattering albedo, and $F_{s}$ is the incoming solar flux. For the Eddington approximation, $P\left(\tau, \mu, \mu^{\prime}\right)=1+3 g(\tau) \mu \mu^{\prime}$ for $-1 \leq \mu \leq 1$. For the scattering atmosphere, the irradiance fluxes in the upward and downward directions can be written as

$$
F^{ \pm}(\tau)=2 \pi \int_{0}^{ \pm 1} I(\tau, \mu) \mu d \mu .
$$

To simulate a realistic medium such as cloud or snow, we consider $\omega(\tau)$ and $g(\tau)$ to vary with $\tau$, and we use exponential expressions here to simplify the process. The single-scattering albedo and asymmetry factor are written as

$$
\begin{gathered}
\omega(\tau)=\hat{\omega}+\varepsilon_{\omega}\left(e^{-a \tau}-e^{-a \tau_{0} / 2}\right), \\
g(\tau)=\hat{g}+\varepsilon_{g}\left(e^{-b \tau}-e^{-b \tau_{0} / 2}\right),
\end{gathered}
$$

where $\tau_{0}$ is the optical depth of the layer, $\hat{\omega}$ is the singlescattering albedo at $\tau_{0} / 2$, and $\hat{g}$ is the asymmetry factor at the same place. Both $\varepsilon_{g}$ and $\varepsilon_{\omega}$ are small parameters that are far less than $\hat{g}$ and $\hat{\omega}$, respectively, in a realistic medium.

By perturbation theory (Kato 1966), the corresponding flux can also be expanded by using the perturbation coefficients $\varepsilon_{\omega}$ and $\varepsilon_{g}$ :

$$
\begin{aligned}
& F^{+}=F_{0}^{+}+\varepsilon_{\omega} F_{1}^{+}+\varepsilon_{g} F_{2}^{+}, \\
& F^{-}=F_{0}^{-}+\varepsilon_{\omega} F_{1}^{-}+\varepsilon_{g} F_{2}^{-} .
\end{aligned}
$$

By using the Eddington approximation, we can obtain differential equations for $F^{+}$and $F^{-}$; a detailed derivation is presented in appendix $A$. These equations [see Eq. (A4) for the precise formulation] can be rewritten as separate equations for $F_{0}^{ \pm}, F_{1}^{ \pm}$, and $F_{2}^{ \pm}$. We obtain the following equations for the scattered flux $F_{0}^{ \pm}$:

$$
\begin{aligned}
\frac{d F_{0}^{+}}{d \tau} & =\gamma_{1}^{0} F_{0}^{+}-\gamma_{2}^{0} F_{0}^{-}-\gamma_{3}^{0} \hat{\omega} F_{s} e^{-\tau / \mu_{0}}, \\
\frac{d F_{0}^{-}}{d \tau} & =\gamma_{2}^{0} F_{0}^{+}-\gamma_{1}^{0} F_{0}^{-}+\gamma_{4}^{0} \hat{\omega} F_{s} e^{-\tau / \mu_{0}}, \\
F_{0}^{-}(\tau=0) & =0 \\
F_{0}^{+}\left(\tau=\tau_{0}\right) & =R_{\mathrm{dif}} F_{0}^{-}\left(\tau=\tau_{0}\right)+R_{\mathrm{dir}} \mu_{0} F_{s} e^{-\tau_{0} / \mu_{0}},
\end{aligned}
$$

where $\gamma_{1,2,3,4}^{0}$ is defined in the appendix A. Equation (5) is the standard RT equation for a homogeneous layer (Meador and Weaver 1980) and has the following solution: 


$$
\begin{aligned}
& F_{0}^{+}=K_{1} e^{k \tau}+\Gamma K_{2} e^{-k \tau}+G_{1} e^{-\tau / \mu_{0}}, \\
& F_{0}^{-}=\Gamma K_{1} e^{k \tau}+K_{2} e^{-k \tau}+G_{2} e^{-\tau / \mu_{0}},
\end{aligned}
$$

where $K_{1}, K_{2}, G_{1}, G_{2}, \Gamma$, and $k$ are defined in appendix B. According to Eq. (A4), the equations for the perturbation terms $F_{i}^{ \pm}(i=1,2)$ are

$$
\begin{aligned}
\frac{d F_{i}^{+}}{d \tau}= & \gamma_{1}^{0} F_{i}^{+}-\gamma_{2}^{0} F_{i}^{-}+\left(e^{-a_{i} \tau}-e^{-a_{i} \tau_{0} / 2}\right)\left(\gamma_{1}^{i} F_{0}^{+}-\gamma_{2}^{i} F_{0}^{-}\right) \\
& -\gamma_{3}^{i-1} F_{s}\left(e^{-a_{i} \tau}-e^{-a_{i} \tau_{0} / 2}\right) e^{-\tau / \mu_{0}}, \\
\frac{d F_{i}^{-}}{d \tau}= & \gamma_{2}^{0} F_{i}^{+}-\gamma_{1}^{0} F_{i}^{-}+\left(e^{-a_{i} \tau}-e^{-a_{i} \tau_{0} / 2}\right)\left(\gamma_{2}^{i} F_{0}^{+}-\gamma_{1}^{i} F_{0}^{-}\right) \\
& +\gamma_{4}^{i-1} F_{s}\left(e^{-a_{i} \tau}-e^{-a_{i} \tau_{0} / 2}\right) e^{-\tau / \mu_{0}}, \\
F_{i}^{-}(\tau= & 0)=0, \quad F_{i}^{+}\left(\tau=\tau_{0}\right)=R_{\mathrm{dif}} F_{i}^{-}\left(\tau=\tau_{0}\right),
\end{aligned}
$$

where $\gamma_{3}^{1}=-\gamma_{4}^{1}=\hat{\omega} \gamma_{3}^{2}, \gamma_{1,2}^{1}, \gamma_{1,2}^{2}$, and $\gamma_{3}^{2}$ are defined in appendix A. The solutions (the details are in appendix C) are

$$
\begin{aligned}
M_{i}= & A_{i}^{+} e^{-k \tau}+B_{i}^{+} e^{k \tau}+P_{i}^{+} e^{-\left(k+a_{i}\right) \tau}+Q_{i}^{+} e^{\left(k-a_{i}\right) \tau} \\
& +R_{i}^{+} e^{-\left(a_{i}+1 / \mu_{0}\right) \tau}-\frac{\eta_{4 i}^{+}}{2 k} e^{-k \tau}+\frac{\eta_{5 i}^{+}}{2 k} e^{k \tau}+\frac{\eta_{6 i}^{+} \mu_{0}^{2}}{1-\mu_{0}^{2} k^{2}} e^{-\tau / \mu_{0}},
\end{aligned}
$$

$$
\begin{aligned}
N_{i}= & C_{i}^{+} e^{-k \tau}+D_{i}^{+} e^{k \tau}+P_{i}^{-} e^{-\left(k+a_{i}\right) \tau}+Q_{i}^{-} e^{\left(k-a_{i}\right) \tau} \\
& +R_{i}^{-} e^{-\left(a_{i}+1 / \mu_{0}\right) \tau}-\frac{\eta_{4 i}^{-}}{2 k} e^{-k \tau}+\frac{\eta_{5 i}^{-}}{2 k} e^{k \tau}+\frac{\eta_{6 i}^{-} \mu_{0}^{2}}{1-\mu_{0}^{2} k^{2}} e^{-\tau / \mu_{0}}
\end{aligned}
$$

Finally, we can obtain $F_{i}^{+}$and $F_{i}^{-}$as

$$
\begin{aligned}
F_{i}^{+}= & D_{1 i}^{+} e^{-k \tau}+D_{2 i}^{+} e^{k \tau}+\varphi_{1 i}^{+} e^{-\left(k+a_{i}\right) \tau}+\varphi_{2 i}^{+} e^{\left(k-a_{i}\right) \tau} \\
& +\varphi_{3 i}^{+} e^{-\left(a_{i}+1 / \mu_{0}\right) \tau}+\varphi_{4 i}^{+} \tau e^{-k \tau}+\varphi_{5 i}^{+} \tau e^{k \tau}+\varphi_{6 i}^{+} e^{-\tau / \mu_{0}}, \\
F_{i}^{-}= & D_{1 i}^{-} e^{-k \tau}+D_{2 i}^{-} e^{k \tau}+\varphi_{1 i}^{-} e^{-\left(k+a_{i}\right) \tau}+\varphi_{2 i}^{-} e^{\left(k-a_{i}\right) \tau} \\
& +\varphi_{3 i}^{-} e^{-\left(a_{i}+1 / \mu_{0}\right) \tau}+\varphi_{4 i}^{-} \tau e^{-k \tau}+\varphi_{5 i}^{-} \tau e^{k \tau}+\varphi_{6 i}^{-} e^{-\tau / \mu_{0}},
\end{aligned}
$$

where $D_{1 i}, D_{2 i}, \varphi_{1 i}, \varphi_{2 i}, \varphi_{3 i}, \varphi_{4 i}, \varphi_{5 i}$, and $\varphi_{6 i}$ are defined in appendix D.

\section{Results and discussion}

\section{a. Idealized medium}

We analyze the accuracy of the new inhomogeneous RT solution by applying it to a single-layer inhomogeneous scattering medium. In the benchmark calculations, the inhomogeneous medium is divided into 100 sublayers, and the IOPs vary from layer to layer to resolve their vertical variation. The Eddington approximation is used in the benchmark calculation. The optical depth of the medium $\tau_{0}$ varies from 0.1 to 50 .
First, we consider the case of vertical variation in single-scattering albedo with $\omega(\tau)=0.9-0.05\left(e^{-a \tau}-\right.$ $\left.e^{-a \tau_{0} / 2}\right)$. The asymmetry factor is constant, with $g(\tau)=0.75$. Different values of $a$ control the degree of vertical variation in $\omega(\tau)$. The results of reflection are shown in the top two rows of Fig. 1 for $a=0.01,0.05$, and 0.25 : the larger the value of $a$, the stronger the vertical variation of $\omega(\tau)$. Figure 1 shows that the reflection calculated with the inhomogeneous solution is always much more accurate than that with the homogeneous solution. For $a=0.01$, the relative error in the reflection with the homogeneous solution is about $5.8 \%$ at $\tau_{0}=50$, whereas the error with the inhomogeneous solution is less than $0.4 \%$. For $a=0.25$, the relative error in the reflection with the homogeneous solution increases to $20 \%$, but it is limited to $4.5 \%$ with the inhomogeneous solution. All the results show that the new algorithm is significantly more accurate than the homogeneous RT solution, especially for large optical depths. There is an interesting phenomenon whereby the reflectance decreases after reaching its maximum. This is because the single-scattering albedo in layers having nearly the same optical depth decreases with the optical depth $\tau_{0}$. The absorptance results are shown in the bottom two rows of Fig. 1. For $a=0.01$, the relative error with the homogeneous RT solution is $2.3 \%$ at $\tau_{0}=50$, whereas the error is less than $0.2 \%$ with the inhomogeneous solution. For $a=0.25$, the relative error with the homogeneous solution increases to $7 \%$, whereas the relative error with the inhomogeneous solution does not exceed $1.5 \%$. All results with the homogeneous solution underestimate the solar absorption.

Second, we consider the case of vertical variation in the asymmetry factor, with $g(\tau)=0.8-0.1\left(e^{-b \tau}-e^{-b \tau_{0} / 2}\right)$, while the single-scattering albedo is constant at $\omega(\tau)=0.99$. Different values of $b$ represent different degrees of vertical variation in $g(\tau)$; we set $b=0.01,0.05$, and 0.25 . The reflection results are shown in the top two rows of Fig. 2. The maximum relative error in refection with the homogeneous solution can be over $4 \%$, whereas the error is not more than $0.6 \%$ with the inhomogeneous solution. The corresponding absorptance results are shown in the bottom two rows of Fig. 2. The maximum relative error with the homogeneous solution can reach $8 \%$, whereas the relative error with the inhomogeneous solution is roughly $1 \%$ at large optical depths. Overall, the inhomogeneous solution can dramatically improve the accuracy in both reflection and absorption from that given by the homogeneous solution.

Third, we examine the sensitivity to the two other parameters, $\varepsilon_{\omega}$ and $\varepsilon_{g}$. In the first and second rows of Fig. 3, we take the case of $g=0.75, \omega(\tau)=0.9+$ $\varepsilon_{\omega}\left(e^{-a \tau}-e^{-a \tau_{0} / 2}\right)$, and $a=0.01$, where $\tau_{0}$ varies from 0.05 

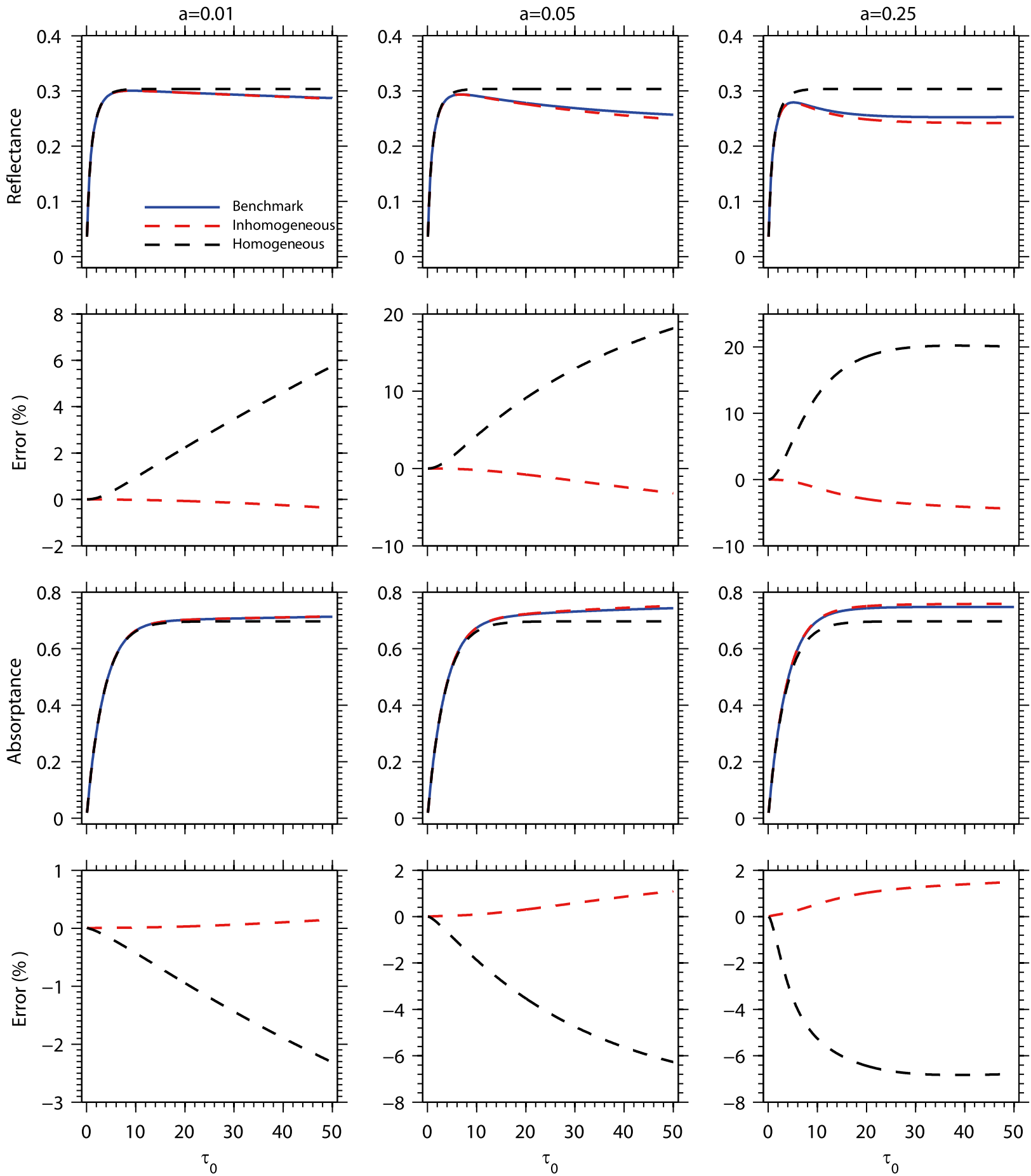

FIG. 1. (first row) Reflectance vs optical depth and (second row) the corresponding relative errors with the inhomogeneous and homogeneous solutions. The asymmetry factor $g$ is held constant, and the single-scattering albedo $\omega$ is a function of optical depth. (third row),(fourth row) As in the first and second rows, resepectively, but for absorptance.

to 50 and $\varepsilon_{\omega}$ varies from -0.2 to -0.002 . For the reflectance (first row), the relative error with the homogeneous solution can be as high as $20 \%$ for large optical depths with large $\varepsilon_{\omega}$ but only around $4 \%$ with the inhomogeneous solution at the same optical depth and value of $\varepsilon_{\omega}$. The same applies to the absorptance (second row): the relative error can be up to $7 \%$ with the homogeneous solution but is limited to $1.5 \%$ with the 

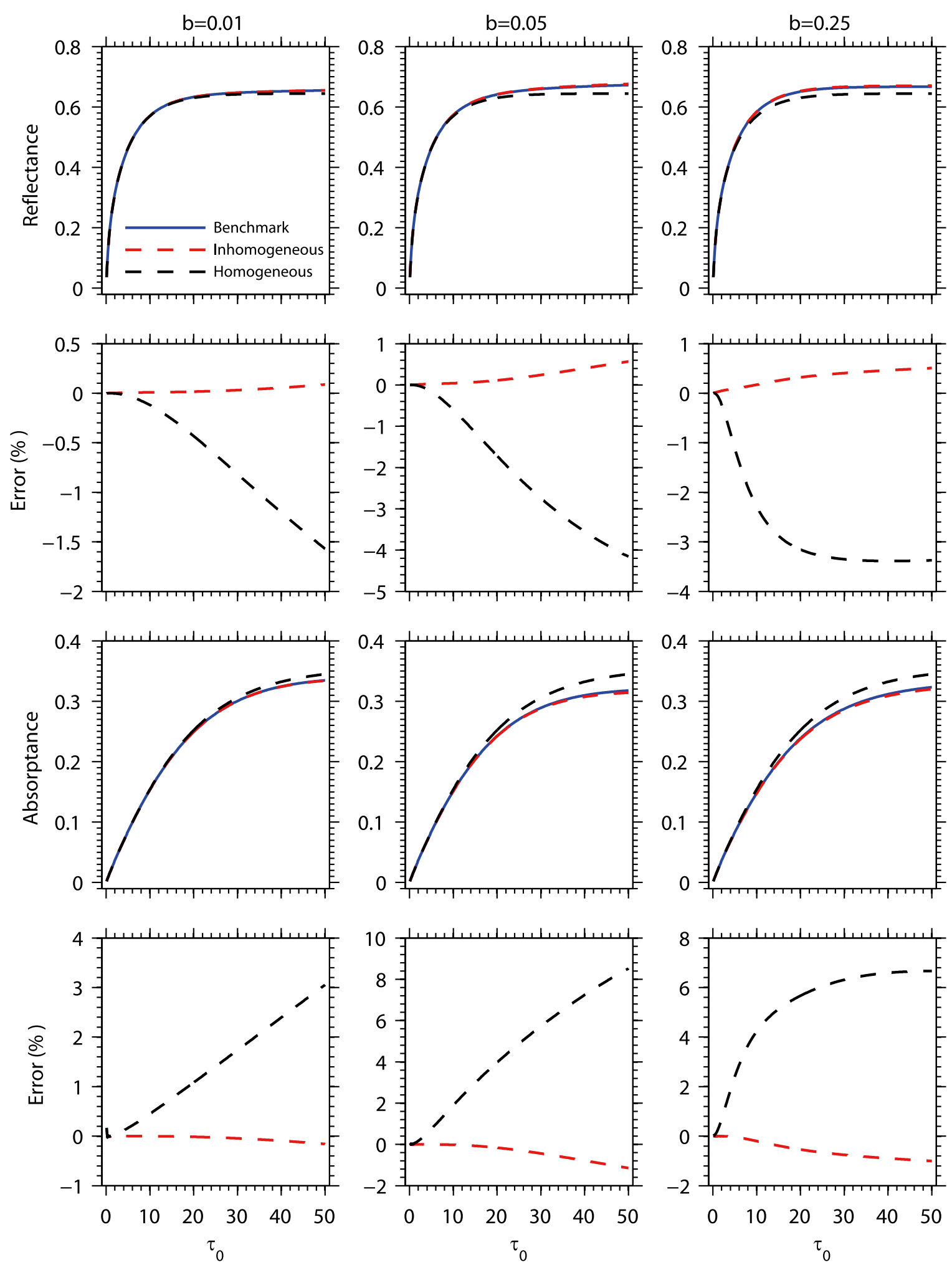

FIG. 2. As in Fig. 1, but the single-scattering albedo $\omega$ is held constant, and the asymmetry factor $g$ is a function of optical depth. 


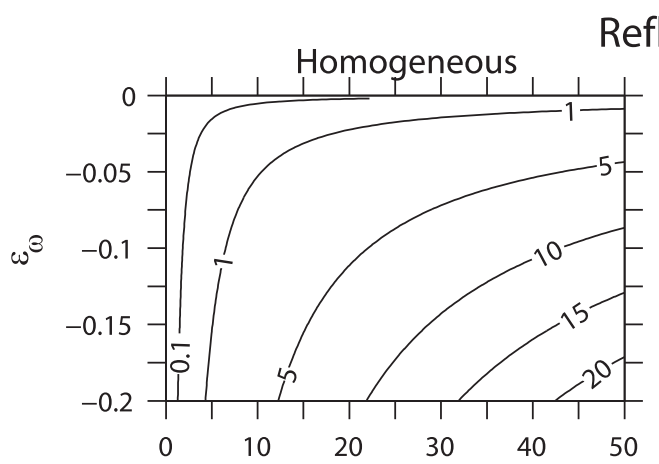

Reflectance
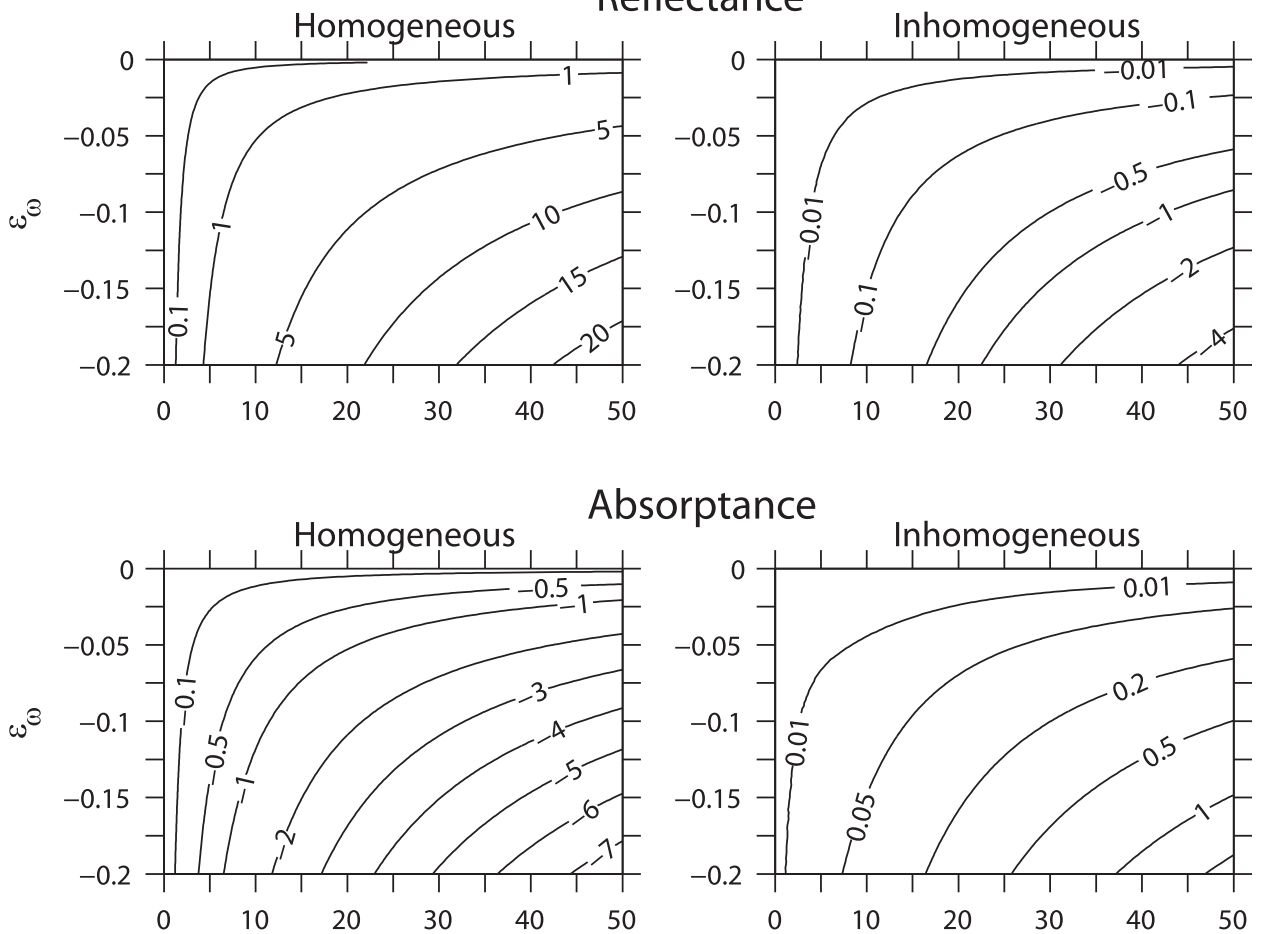

Absorptance

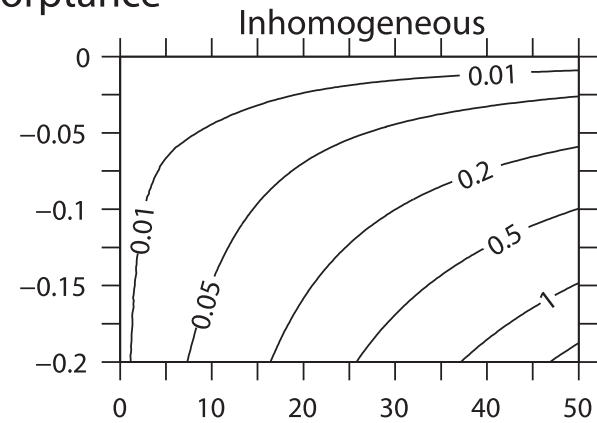

Reflectance
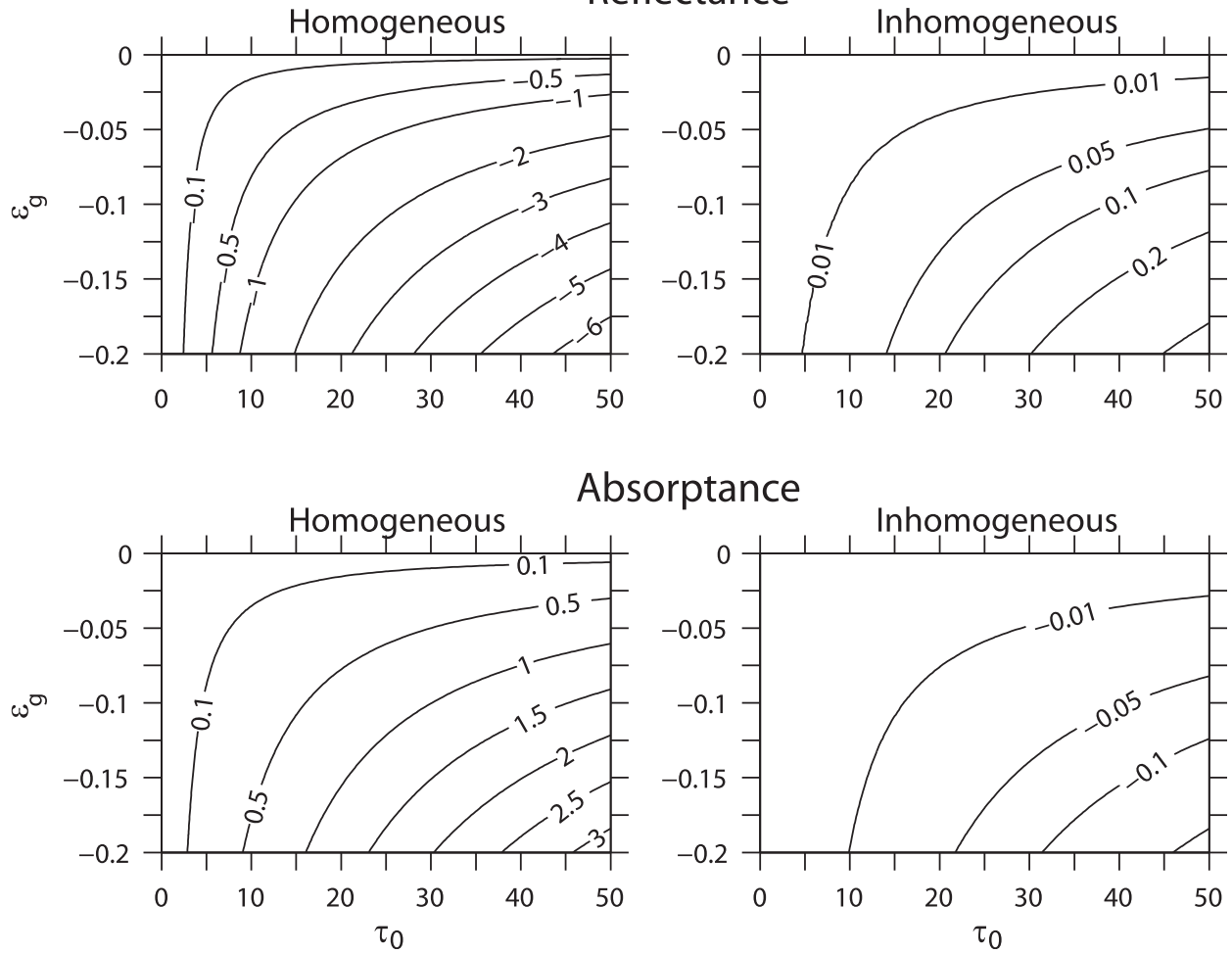

Absorptance

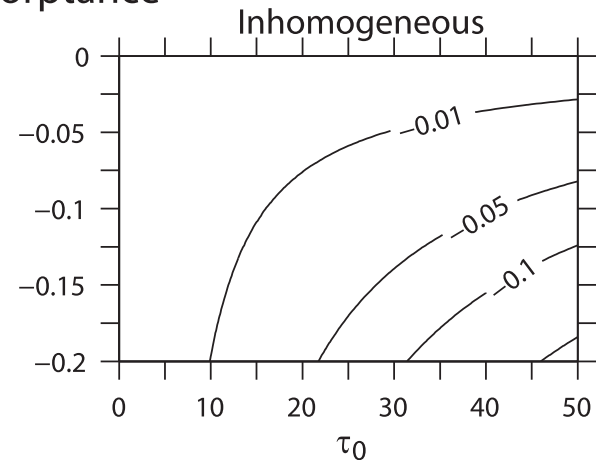

FIG. 3. Relative errors with the (left) homogeneous solution and (right) inhomogeneous solution for the (first row),(third row) reflectance and (second row),(fourth row) absorptance vs $\varepsilon_{\omega}, \varepsilon_{g}$, and optical depth $\tau_{0}$. 
inhomogeneous solution. The accuracy is much higher with the inhomogeneous solution than with the homogeneous solution.

In the third and fourth rows of Fig. 3, we take the case of $\omega=0.9, g(\tau)=0.75+\varepsilon_{g}\left(e^{-b \tau}-e^{-b \tau_{0} / 2}\right)$, and $b=0.01$, where $\tau_{0}$ varies from 0.05 to 50 and $\varepsilon_{g}$ varies from -0.2 to -0.002 . The relative errors with the inhomogeneous solution are more than one order of magnitude smaller than those with the homogeneous solution for both reflectance and absorptance. The relative errors in reflectance (Fig. 3, third row) with the homogeneous solution are greater than $0.5 \%$, whereas with the inhomogeneous solution, they are less than $0.3 \%$ in most regions. The relative errors in absorptance (Fig. 3, fourth row) with the inhomogeneous solution are less than $0.1 \%$ in most regions, whereas with the homogeneous solution, they are greater than $0.1 \%$.

\section{b. Cloud}

The optical properties of ice clouds depend not only on the effective size but also on the complex particle habits (Letu et al. 2012, 2016; Yang et al. 2015). Thus, the ice cloud is not to be considered in this study. We just discuss the water cloud. Observations show that the cloud LWC and droplet radius tend to increase with height above the cloud base (Noonkester 1984). This phenomenon is attributed to the process of adiabatic water vapor condensation. To take into account the internal inhomogeneity of cloud optical properties, we assume that LWC $\left(\mathrm{g} \mathrm{m}^{-3}\right)$ and droplet cross-sectional area (DCA; $\mathrm{cm}^{-2} \mathrm{~m}^{-3}$ ) increase linearly from the cloud base $(0 \mathrm{~m})$ to a position near the cloud top $(1000 \mathrm{~m})$ :

$$
\begin{aligned}
& \mathrm{LWC}=0.22+0.00008 z, \\
& \mathrm{DCA}=100+z,
\end{aligned}
$$

where $0<z<z_{0}, z$ is the height in meters from the cloud base, and $z_{0}$ is the height of the cloud top. We can obtain the cloud effective radius $r_{e}(\mu \mathrm{m})$ from the given LWC and DCA:

$$
r_{e}(z)=\frac{3}{4 \rho} \frac{\mathrm{LWC}}{\mathrm{DCA}} 10^{10},
$$

where $\rho\left(\mathrm{g} \mathrm{m}^{-3}\right)$ is the liquid water density. Therefore, the LWC varies from 0.22 to $0.30 \mathrm{~g} \mathrm{~m}^{-3}$, and $r_{e}$ varies from 2.06 to $16.50 \mu \mathrm{m}$. This is consistent with observations (Chen et al. 2008). The liquid water path (LWP) is defined as LWP $=\int_{0}^{z_{0}} \mathrm{LWC} d z$. In this case, the LWP is $260 \mathrm{~g} \mathrm{~m}^{-2}$, which represents low cloud (Fu et al. 1997). In the benchmark calculations, $z_{0}$ is divided into 100 internal homogeneous sublayers, although other numbers can be chosen (e.g., 200). In principle, more internal sublayers should result in more accurate results. We use 100 internal sublayers throughout this study because having any more makes little difference to the calculated results. Using 100 sublayers is sufficiently accurate to resolve the vertical internal inhomogeneity of the medium. We use the optical properties of a water cloud in the spectral band of $0.25-0.69 \mu \mathrm{m}$ and at $0.94 \mu \mathrm{m}$. We choose the $0.25-0.69-\mu \mathrm{m}$ band because it contains roughly $46 \%$ of the solar radiation energy; the $0.94-\mu \mathrm{m}$ wavelength is at the center of channel 19 of the Moderate Resolution Imaging Spectroradiometer (MODIS), which is used in this paper to illustrate the effect of vertical internal inhomogeneity related to remote sensing. The surface albedo is set to zero. We calculate the asymmetry factor and single-scattering albedo of each sublayer as the benchmark values, which we then use in Eq. (3) to obtain the relevant parameters.

In Figs. $4 \mathrm{a}$ and $4 \mathrm{~b}$, the benchmark values of the inhomogeneous IOPs and the parameterized results for the spectral band of $0.25-0.69 \mu \mathrm{m}$ are shown. The parameterized inhomogeneous IOPs are $1-\omega(\tau)=3.979 \times 10^{-7}$ $-1.897 \times 10^{-6}\left(e^{-0.1539 \tau}-e^{-0.1539 \tau_{0} / 2}\right)$ and $g(\tau)=0.8359$ $+0.0289\left(e^{-0.1539 \tau}-e^{-0.1539 \tau_{0} / 2}\right)$, where $\tau_{0}=110.84$. The corresponding results for reflection and absorption are shown in Figs. 4c-f. For reflection, the relative error with the homogeneous solution increases from $0.25 \%$ to $0.71 \%$ as $\mu_{0}$ increases from 0.01 to 1 , whereas the relative error with the inhomogeneous solution increases from $0.05 \%$ to $0.14 \%$. For absorption, the relative error is not sensitive to $\mu_{0}$; it is around $7.4 \%$ with the homogeneous solution but around only $1.4 \%$ with the inhomogeneous solution.

In Figs. 5a and 5b, the benchmark values of the inhomogeneous IOPs and the parameterized results for the wavelength $0.94 \mu \mathrm{m}$ are shown. The parameterized inhomogeneous IOPs are $1-\omega(\tau)=1.936 \times 10^{-4}-$ $5.263 \times 10^{-4}\left(e^{-0.0357 \tau}-e^{-0.0357 \tau_{0} / 2}\right)$ and $g(\tau)=0.8321-$ $0.0403\left(e^{0.0218 \tau}-e^{0.0218 \tau_{0} / 2}\right)$, where $\tau_{0}=54.46$. For both $g(\tau)$ and $1-\omega(\tau)$, the parameterized results match well with the corresponding benchmark values. Figures $5 \mathrm{c}-\mathrm{f}$ show the corresponding results for reflection and absorption. For reflection, the relative error with the homogeneous solution increases from $1.1 \%$ to $3.0 \%$ as $\mu_{0}$ increases from 0.01 to 1 , whereas the relative error with the inhomogeneous solution increases from $0.7 \%$ to $2.0 \%$. For absorption, the relative error is not sensitive to $\mu_{0}$; it is around $10 \%$ with the homogeneous solution but around only $5.7 \%$ with the inhomogeneous solution.

\section{c. Snow}

Generally, the effective snow grain size $r_{e}(\mu \mathrm{m})$ and the equivalent depth of liquid water in a snowpack $L\left(\mathrm{~g} \mathrm{~cm}^{-2}\right)$ can be assumed to increase linearly with depth: 

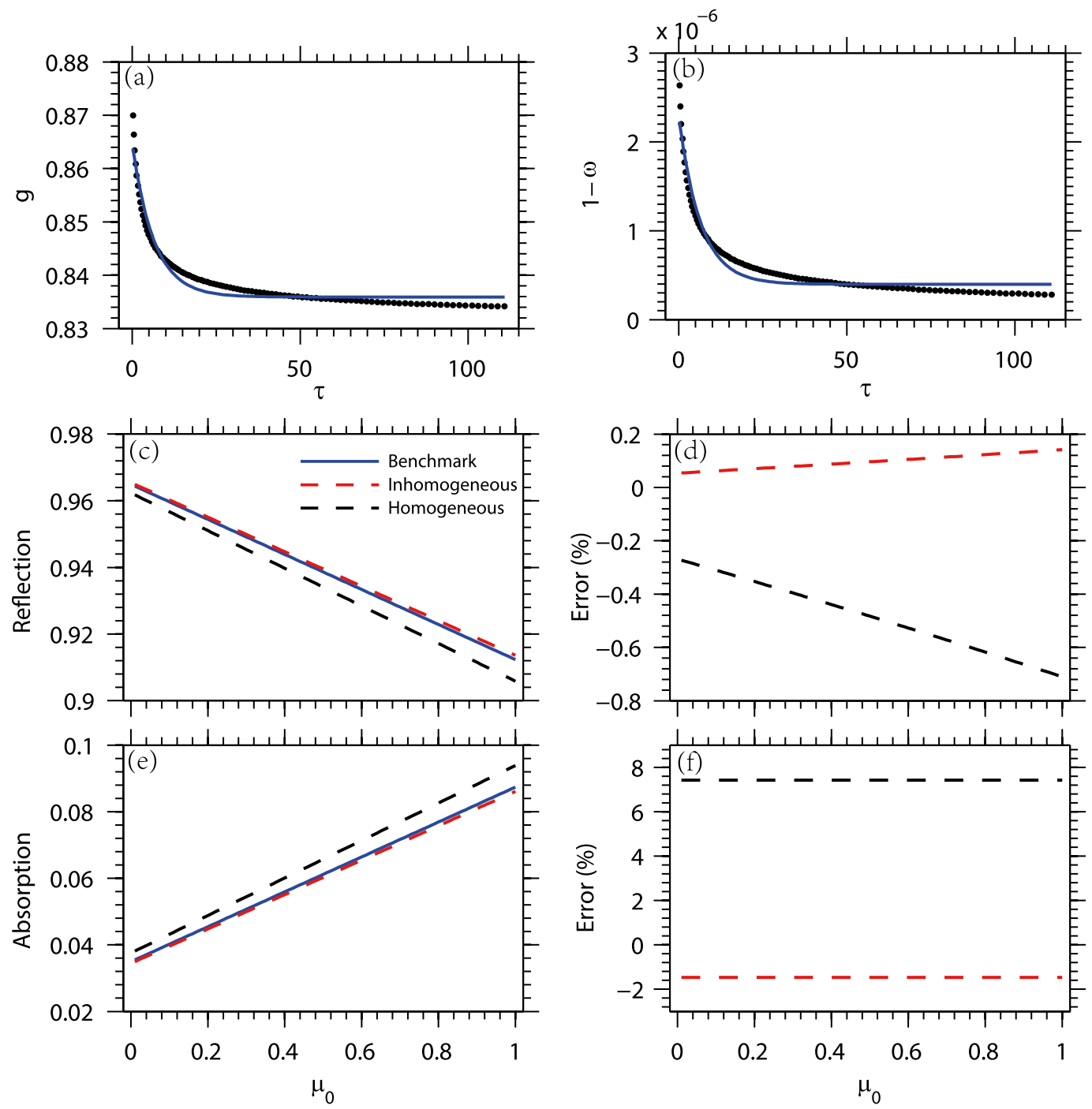

FIG. 4. For the band of 0.25-0.69 $\mu \mathrm{m}$, (a) cloud asymmetry factor and (b) single-scattering albedo vs cloud optical depth; (c) reflectance and (e) absorptance vs optical depth; and (d),(f) relative errors with the inhomogeneous and homogeneous solutions.

$$
\begin{aligned}
& r_{e}=50+5 h, \\
& L=\rho_{\text {snow }} h,
\end{aligned}
$$

where $h$ is the snow thickness in centimeters from the snow top $\left(0<h<h_{0}\right.$, with $h_{0}$ the thickness of the whole snow layer) and $\rho_{\text {snow }}=0.15 \mathrm{~g} \mathrm{~cm}^{-3}$ is the density of snow. We assume $h_{0}$ is equal to $100 \mathrm{~cm}$; thus, the effective size of snow varies from 50 to $550 \mu \mathrm{m}$. The snow layer is divided into 100 internal homogeneous sublayers in the benchmark calculations.

We focus on reflection and absorption by snow in the spectral band of $0.25-0.69 \mu \mathrm{m}$ and at the wavelength $0.94 \mu \mathrm{m}$. The surface albedo is set at zero. We calculate the asymmetry factor and single-scattering albedo of each sublayer as the benchmark values (Wiscombe and
Warren 1980) and then use these in Eq. (3) to obtain the relevant parameters.

Figures $6 \mathrm{a}$ and $6 \mathrm{~b}$ show the benchmark values of the inhomogeneous IOPs and the parameterized results for the spectral band $0.25-0.69 \mu \mathrm{m}$. The parameterized inhomogeneous IOPs are $g(\tau)=0.8896-$ $0.0061\left(e^{-0.0039 \tau}-e^{-0.0039 \tau_{0} / 2}\right) \quad$ and $\omega(\tau)=0.99998-$ $0.0000039\left(e^{0.0040 \tau}-e^{0.0040 \tau_{0} / 2}\right), \quad$ with $\quad \tau_{0}=776.31$. Figures $6 \mathrm{c}-\mathrm{f}$ show the results for reflection and absorption with the homogeneous and inhomogeneous solutions and their relative errors against the benchmark calculations. For reflection, the relative error with the homogeneous solution is $0.8 \%$ for $\mu_{0}=0.01$, rising to $1.9 \%$ for $\mu_{0}=1$. The error is reduced by using the inhomogeneous solution, with the relative error increasing 

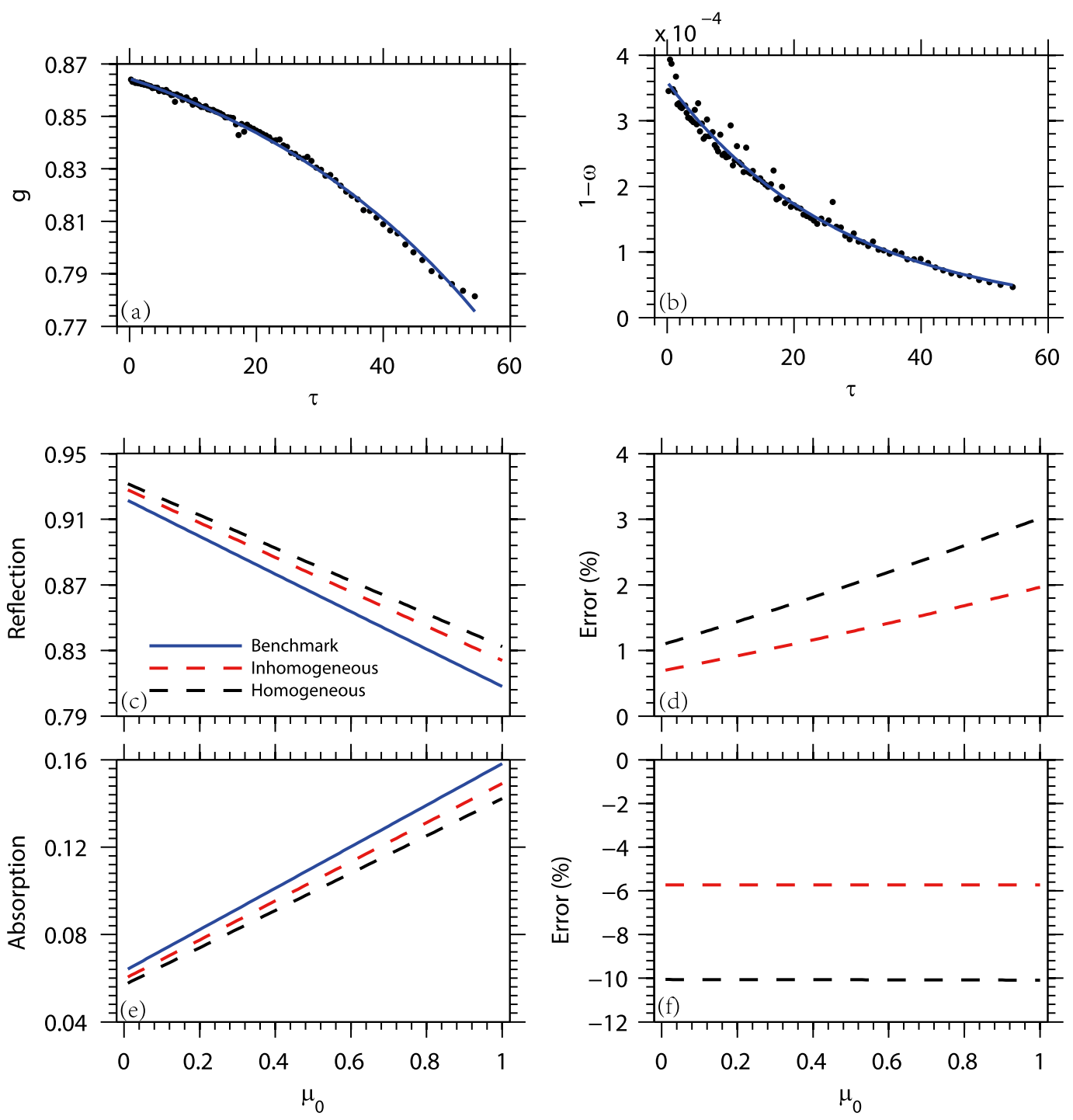

FIG. 5. As in Fig. 4, but for the wavelength $0.94 \mu \mathrm{m}$.

from only $0.4 \%$ to only $1.0 \%$. For absorption, the relative error with the homogeneous solution can be in excess of $72 \%$, whereas it is no more than $40 \%$ with the inhomogeneous solution. However, because the absorption is very small in this case, the absolute error with the inhomogeneous solution is less than 0.008 .

Figures $7 \mathrm{a}$ and $7 \mathrm{~b}$ show the benchmark values of the inhomogeneous IOPs and the parameterized results for the wavelength $0.94 \mu \mathrm{m}$. The parameterized inhomogeneous IOPs are $g(\tau)=0.8932-0.0117\left(e^{-0.0032 \tau}-\right.$ $\left.e^{-0.0032 \tau_{0} / 2}\right) \quad$ and $\quad \omega(\tau)=0.9987-0.00028\left(e^{0.0040 \tau}-\right.$ $\left.e^{0.0040 \tau_{0} / 2}\right)$, with $\tau_{0}=779.08$. Figures $7 \mathrm{c}-\mathrm{f}$ show the results for reflection and absorption with the homogeneous and inhomogeneous solutions. For reflection, the relative error with the homogeneous solution is $10 \%$ for $\mu_{0}=0$, rising to $27.5 \%$ for $\mu_{0}=1$. The inhomogeneous solution produces smaller relative errors, ranging from $1.2 \%$ to $3.7 \%$. For absorption, the relative error with the homogeneous solution can be in excess of $120 \%$, whereas the relative error with the inhomogeneous solution is not more than $15 \%$. Although the relative error with the inhomogeneous solution can be as high as $15 \%$, the absorption is very small in this case, with the absolute error with the inhomogeneous solution being less than 0.04 .

\section{Summary and conclusions}

In the above, we have considered the vertically inhomogeneous structures of only cloud and snow, whereas all physical quantities in the atmosphere are vertically inhomogeneous (e.g., the concentrations of all types of gases and aerosols). In current climate models, 

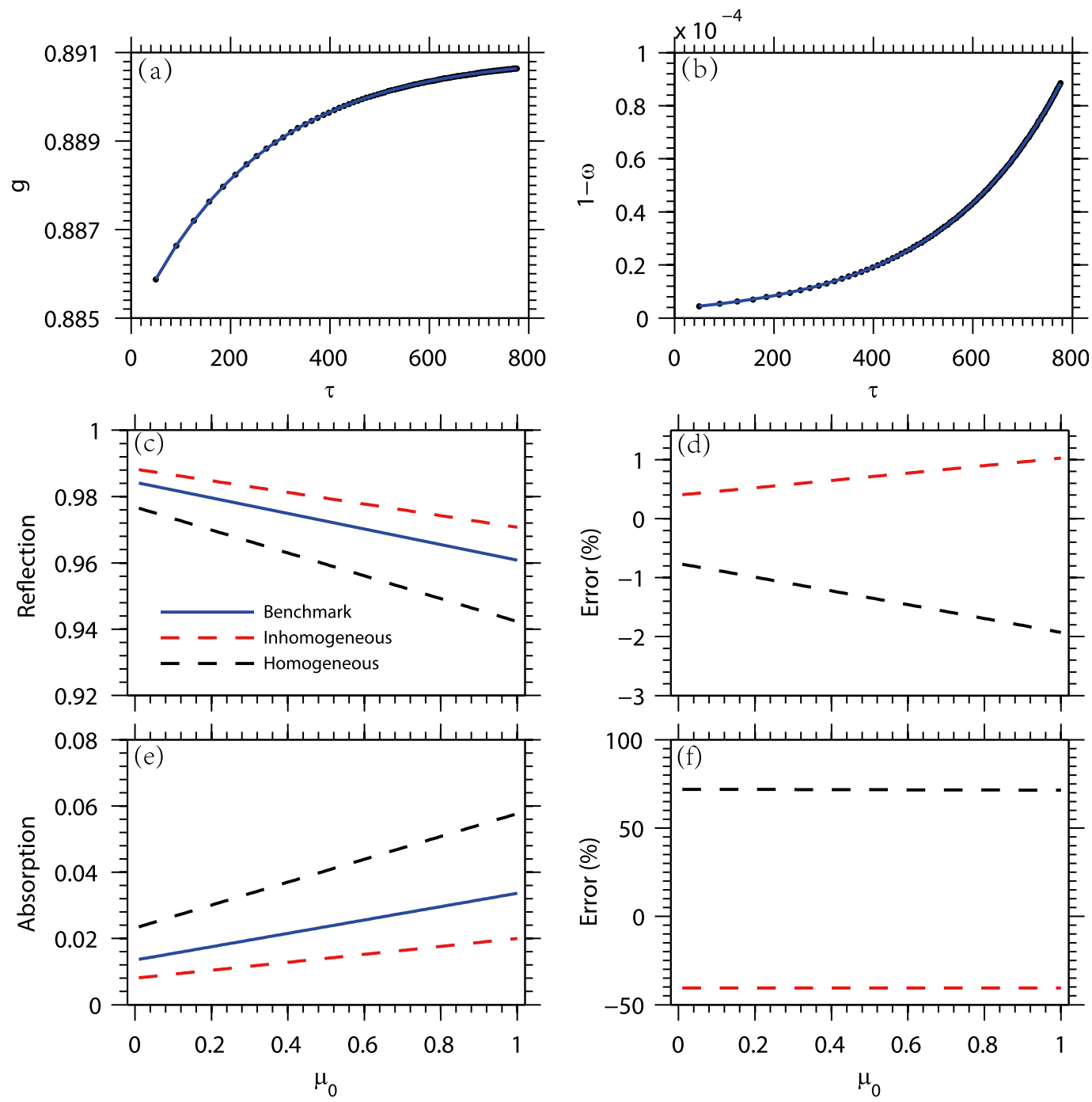

FIG. 6. For the band of $0.25-0.69 \mu \mathrm{m}$, (a) snow asymmetry factor (b) single-scattering albedo vs cloud optical depth; (c) reflectance and (e) absorptance vs optical depth; and (d),(f) relative errors with the inhomogeneous and homogeneous solutions.

the vertical-layer resolution is far from that required to resolve such vertical inhomogeneity. In this study, we have proposed a new inhomogeneous RT solution to address the vertical inhomogeneity by introducing an internal variation of IOPs inside each model layer. This scheme is based on standard perturbation theory and allows us to use the standard RT solution for homogeneous layers to identify a zeroth-order equation and a first-order equation that includes the inhomogeneous effect. The new RT solution can accurately express the inhomogeneous effect in each model layer, and it reduces to the standard Eddington solution when the medium is homogeneous.

The accuracy of the new inhomogeneous RT solution has been investigated systematically for both an idealized medium and realistic samples of cloud and snow. For the idealized medium, the relative errors in reflection and absorption calculated with the inhomogeneous RT solution are significantly smaller than those obtained with the standard homogeneous RT solution. Generally, the relative error with the homogeneous RT solution increases with optical depth, and the relative error can be over $20 \%$. However, the relative error with the inhomogeneous RT solution is not particularly sensitive to optical depth, and the relative error is limited to $4 \%$ in most cases.

The new inhomogeneous RT solution is a good way to resolve cloud vertical inhomogeneity. In the spectral band of $0.25-0.69 \mu \mathrm{m}$, the relative error in the inhomogeneous RT solution is no more than $1.4 \%$, 

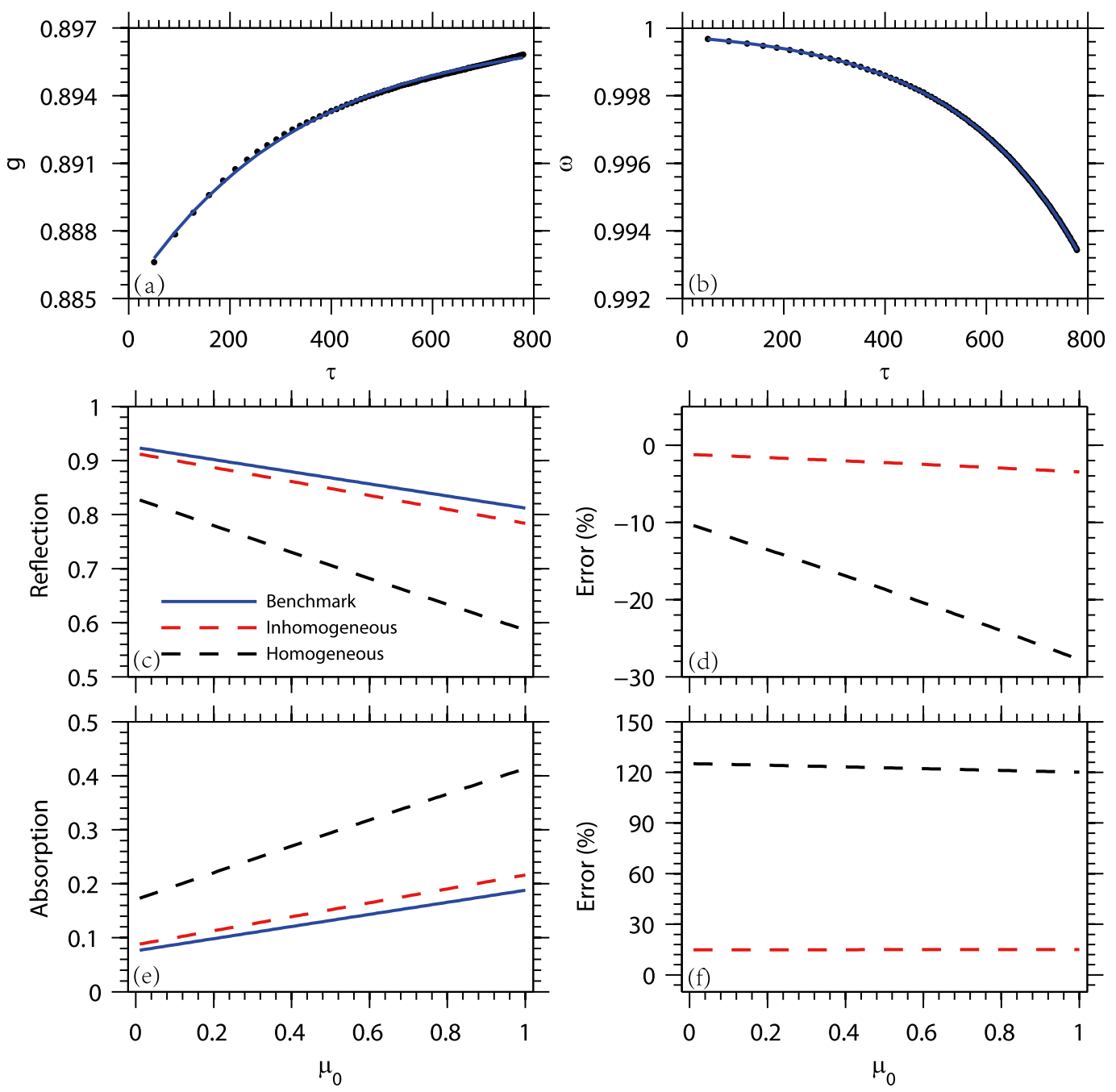

FIG. 7. As in Fig. 6, but for the wavelength $0.94 \mu \mathrm{m}$.

whereas the error with the homogeneous RT solution can be up to $7.4 \%$. At the specific wavelength of $0.94 \mu \mathrm{m}$, the relative error with the inhomogeneous solution is not more than $5.7 \%$ but can be up to $10 \%$ with the homogeneous solution.

The new inhomogeneous RT solution can also resolve snow vertical inhomogeneity. In the spectral band of $0.25-0.69 \mu \mathrm{m}$, the relative error in reflection with the homogeneous solution can be up to $72 \%$, but the error is reduced to less than $40 \%$ by using the inhomogeneous solution. At the specific wavelength of $0.94 \mu \mathrm{m}$, the relative error in the inhomogeneous solution is not more than $15 \%$ but can be up to $120 \%$ in the homogeneous solution.

In specific spectral bands or at specific wavelengths, the vertical variations in IOPs can typically be fitted easily into Eq. (3) to obtain the required parameters. A simple fitting program can be easily incorporated into a climate model to produce the inhomogeneous IOPs of stratocumulus clouds. If no such cloud-inhomogeneity information is available in the current climate models, the vertical variation rates of cloud LWC and DCA can be derived empirically from observations, which show that the vertical variation rates of LWC and DCA in stratocumulus clouds are not very different (Vane et al. 2006; Boutle et al. 2014; Young et al. 2013; Luo et al. 2014; Zhang et al. 2017). For snow, the current climate models typically can calculate the snow age (Namazi et al. 2015). In principle, the vertical variation information for snow could be obtained to fit the IOPs in Eq. (3).

In this study, we presented only a single-layer inhomogeneous RT solution, which corresponds to the standard Eddington-approximation solution. To implement the new solution in a climate model, the process of adding layer-to-layer connections has to be resolved 
(Hansen 1971; de Haan et al. 1987; Nakajima et al. 2000; Zhang et al. 2013; Zhang and Li 2013). Under the homogeneous condition, the single-layer result in reflection and transmission is the same for an upward path and a downward path, but this is not true for an inhomogeneous layer. Therefore, the adding process has to be modified. We will present an algorithm for this multilayer adding process in our next study, in which the climatic impact of inhomogeneous clouds and inhomogeneous snows will be explored. The code base for the inhomogeneous RT solution is available from the authors upon request.

Acknowledgments. This work is supported by the National Natural Science Foundation of China (41675003, 41675056, and 91537213), a Grant-in-Aid for Scientific Research (16F16031) from the Japan Society for the Promotion of Science, the Natural Science Foundation of Jiangsu Province (BK20150931), and the Priority Academic Program Development of Jiangsu Higher Education Institutions (PAPD). The ESMC contribution number is ESMC 180.

\section{APPENDIX A}

\section{Perturbation Equations}

According to the Eddington approximation, the radiative intensity $I(\tau, \mu)$ can be written as

$$
I(\tau, \mu)=I_{0}(\tau)+I_{1}(\tau) \mu, \quad-1 \leq \mu \leq 1 .
$$

Using Eqs. (1), (2), and (A1), we obtain $\frac{d F^{+}(\tau)}{d \tau}=\gamma_{1}(\tau) F^{+}(\tau)-\gamma_{2}(\tau) F^{-}(\tau)-\gamma_{3}(\tau) \omega(\tau) F_{s} e^{-\tau / \mu_{0}}$,

$$
\begin{aligned}
& \frac{d F^{-}(\tau)}{d \tau}= \gamma_{2}(\tau) F^{+}(\tau)-\gamma_{1}(\tau) F^{-}(\tau) \\
&+\left[1-\gamma_{3}(\tau)\right] \omega(\tau) F_{s} e^{-\tau / \mu_{0}} \\
& F^{-}(\tau=0)=0 \\
& F^{+}\left(\tau=\tau_{0}\right)=R_{\mathrm{dif}} F^{-}\left(\tau=\tau_{0}\right)+R_{\mathrm{dir}} \mu_{0} F_{s} e^{-\tau_{0} / \mu_{0}}
\end{aligned}
$$

where $\gamma_{1}(\tau)=(1 / 4)\{7-[4+3 g(\tau)] \omega(\tau)\}, \gamma_{2}(\tau)=-(1 / 4)$ $\{1-[4-3 g(\tau)] \omega(\tau)\}$, and $\gamma_{3}(\tau)=(1 / 4)\left[2-3 g(\tau) \mu_{0}\right]$ $\tau_{0}$ is the optical depth of the single layer; and $R_{\text {dif }}\left(R_{\text {dir }}\right)$ is the diffuse (direct) reflection from the layer below or the diffuse (direct) surface albedo.

Substituting $\gamma_{1}, \gamma_{2}$, and $\gamma_{3}$ into Eq. (3) and ignoring the small second-order parameters $\varepsilon_{g}^{2}, \varepsilon_{\omega}^{2}$, and $\varepsilon_{g} \varepsilon_{\omega}$, we get

$\gamma_{1}(\tau)=\gamma_{1}^{0}+\gamma_{1}^{1} \varepsilon_{\omega}\left(e^{-a \tau}-e^{-a \tau_{0} / 2}\right)+\gamma_{1}^{2} \varepsilon_{g}\left(e^{-b \tau}-e^{-b \tau_{0} / 2}\right)$,

$\gamma_{2}(\tau)=\gamma_{2}^{0}+\gamma_{2}^{1} \varepsilon_{\omega}\left(e^{-a \tau}-e^{-a \tau_{0} / 2}\right)+\gamma_{2}^{2} \varepsilon_{g}\left(e^{-b \tau}-e^{-b \tau_{0} / 2}\right)$,

$\gamma_{3}(\tau)=\gamma_{3}^{0}+\gamma_{3}^{2} \varepsilon_{g}\left(e^{-b \tau}-e^{-b \tau_{0} / 2}\right)$,

where $\quad \gamma_{1}^{0}=(1 / 4)[7-(4+3 \hat{g}) \hat{\omega}], \quad \gamma_{2}^{0}=-(1 / 4)[1-(4-$ $3 \hat{g}) \hat{\omega}], \quad \gamma_{3}^{0}=(1 / 4)\left(2-3 \hat{g} \mu_{0}\right), \quad \gamma_{1}^{1}=-(1 / 4)(4+3 \hat{g})$, $\gamma_{1}^{2}=-(3 / 4) \hat{\omega}, \quad \gamma_{2}^{1}=(1 / 4)(4-3 \hat{g}), \quad \gamma_{2}^{2}=-(3 / 4) \hat{\omega}, \quad$ and $\gamma_{3}^{2}=-(3 / 4) \mu_{0}$.

Substituting Eqs. (4) and (A3) into Eq. (A2) yields

$$
\begin{aligned}
\frac{d F^{+}}{d \tau}= & {\left[\gamma_{1}^{0}+\gamma_{1}^{1} \varepsilon_{\omega}\left(e^{-a \tau}-e^{-a \tau_{0} / 2}\right)+\gamma_{1}^{2} \varepsilon_{g}\left(e^{-b \tau}-e^{-b \tau_{0} / 2}\right)\right]\left(F_{0}^{+}+\varepsilon_{\omega} F_{1}^{+}+\varepsilon_{g} F_{2}^{+}\right)-\left[\gamma_{2}^{0}+\gamma_{2}^{1} \varepsilon_{\omega}\left(e^{-a \tau}-e^{-a \tau_{0} / 2}\right)\right.} \\
& \left.+\gamma_{2}^{2} \varepsilon_{g}\left(e^{-b \tau}-e^{-b \tau_{0} / 2}\right)\right]\left(F_{0}^{-}+\varepsilon_{\omega} F_{1}^{-}+\varepsilon_{g} F_{2}^{-}\right)-\left[\hat{\omega} \gamma_{3}^{0}+\gamma_{3}^{0} \varepsilon_{\omega}\left(e^{-a \tau}-e^{-a \tau_{0} / 2}\right)+\hat{\omega} \gamma_{3}^{2} \varepsilon_{g}\left(e^{-b \tau}-e^{-b \tau_{0} / 2}\right)\right] F_{s} e^{-\tau / \mu_{0}}, \\
\frac{d F^{-}}{d \tau}= & {\left[\gamma_{2}^{0}+\gamma_{2}^{1} \varepsilon_{\omega}\left(e^{-a \tau}-e^{-a \tau_{0} / 2}\right)+\gamma_{2}^{2} \varepsilon_{g}\left(e^{-b \tau}-e^{-b \tau_{0} / 2}\right)\right]\left(F_{0}^{+}+\varepsilon_{\omega} F_{1}^{+}+\varepsilon_{g} F_{2}^{+}\right)-\left[\gamma_{1}^{0}+\gamma_{1}^{1} \varepsilon_{\omega}\left(e^{-a \tau}-e^{-a \tau_{0} / 2}\right)\right.} \\
& \left.+\gamma_{1}^{2} \varepsilon_{g}\left(e^{-b \tau}-e^{-b \tau_{0} / 2}\right)\right]\left(F_{0}^{-}+\varepsilon_{\omega} F_{1}^{-}+\varepsilon_{g} F_{2}^{-}\right)+\left[\hat{\omega} \gamma_{4}^{0}+\gamma_{4}^{0} \varepsilon_{\omega}\left(e^{-a \tau}-e^{-a \tau_{0} / 2}\right)-\hat{\omega} \gamma_{3}^{2} \varepsilon_{g}\left(e^{-b \tau}-e^{-b \tau_{0} / 2}\right)\right] F_{s} e^{-\tau / \mu_{0}},
\end{aligned}
$$

where $\gamma_{4}^{0}=\left(1-\gamma_{3}^{0}\right)$. 


\section{APPENDIX B}

\section{Coefficients of Eq. (6)}

The terms $K_{1}, K_{2}, G_{1}, G_{2}, \Gamma$, and $k$ in Eq. (6) are

$$
\begin{aligned}
& K_{1}=\frac{\left(\Gamma-R_{\mathrm{dif}}\right) \Gamma G_{2} e^{-k \tau_{0}}-\left(G_{1}-R_{\mathrm{dif}} G_{2}-R_{\mathrm{dir}} \mu_{0} F_{s}\right) e^{-\tau_{0} / \mu_{0}}}{\left(1-R_{\mathrm{dif}} \Gamma\right) e^{k \tau_{0}-\left(\Gamma-R_{\mathrm{dif}}\right) \Gamma e^{-k \tau_{0}}},}, \\
& K_{2}=\frac{\left(G_{1}-R_{\mathrm{dif}} G_{2}-R_{\mathrm{dir}} \mu_{0} F_{s}\right) e^{-\tau_{0} / \mu_{0}-\left(1-R_{\mathrm{dif}}\right) \Gamma G_{2} e^{-k \tau_{0}}}}{\left(1-R_{\mathrm{dif}} \Gamma\right) e^{k \tau_{0}}-\left(\Gamma-R_{\mathrm{dif}}\right) \Gamma e^{-k \tau_{0}}}, \quad G_{1}=\left[\gamma_{3}^{0}\left(\frac{1}{\mu_{0}}-\gamma_{1}^{0}\right)-\gamma_{2}^{0} \gamma_{4}^{0}\right] \frac{\mu_{0}^{2} \hat{\omega} F_{0}}{1-\mu_{0}^{2} k^{2}}, \\
& G_{2}=-\left[\gamma_{4}^{0}\left(\frac{1}{\mu_{0}}+\gamma_{1}^{0}\right)+\gamma_{2}^{0} \gamma_{3}^{0}\right] \frac{\mu_{0}^{2} \hat{\omega} F_{0}}{1-\mu_{0}^{2} k^{2}}, \quad \Gamma=1-\frac{2 k}{\gamma_{1}^{0}+\gamma_{2}^{0}+k}, \quad \text { and } \quad k^{2}=\left(\gamma_{1}^{0}+\gamma_{2}^{0}\right)\left(\gamma_{1}^{0}-\gamma_{2}^{0}\right) .
\end{aligned}
$$

\section{APPENDIX C}

\section{Solution of Eq. (7)}

Letting $M_{i}=F_{i}^{+}+F_{i}^{-}$and $N_{i}=F_{i}^{+}-F_{i}^{-}$, Eqs. (7a) and $(7 \mathrm{~b})$ yield

$$
\begin{aligned}
\frac{d M_{i}}{d \tau}= & \left(\gamma_{1}^{0}+\gamma_{2}^{0}\right) N_{i}+\left(\Psi_{i}^{+}+\Psi_{i}^{-}\right) e^{-\left(k+a_{i}\right) \tau}+\left(\zeta_{i}^{+}+\zeta_{i}^{-}\right) e^{\left(k-a_{i}\right) \tau} \\
& +\left(\chi_{i}^{+}+\chi_{i}^{-}\right) e^{-\left(a_{i}+1 / \mu_{0}\right) \tau}-e^{-a_{i} \tau_{0} / 2}\left[\left(\Psi_{i}^{+}+\Psi_{i}^{-}\right) e^{-k \tau}\right. \\
& \left.+\left(\zeta_{i}^{+}+\zeta_{i}^{-}\right) e^{k \tau}+\left(\chi_{i}^{+}+\chi_{i}^{-}\right) e^{-\tau / \mu_{0}}\right], \\
\frac{d N_{i}}{d \tau}= & \left(\gamma_{1}^{0}-\gamma_{2}^{0}\right) M_{i}+\left(\Psi_{i}^{+}-\Psi_{i}^{-}\right) e^{-\left(k+a_{i}\right) \tau}+\left(\zeta_{i}^{+}-\zeta_{i}^{-}\right) e^{\left(k-a_{i}\right) \tau} \\
& +\left(\chi_{i^{+}}-\chi_{i}^{-}\right) e^{-\left(a_{i}+1 / \mu_{0}\right) \tau}-e^{-a_{i} \tau_{0} / 2}\left[\left(\Psi_{i}^{+}-\Psi_{i}^{-}\right) e^{-k \tau}\right. \\
& \left.+\left(\zeta_{i}^{+}-\zeta_{i}^{-}\right) e^{k \tau}+\left(\chi_{i}^{+}-\chi_{i}^{-}\right) e^{-\tau / \mu_{0}}\right],
\end{aligned}
$$

where $\Psi_{i}^{+}=K_{2}\left(\gamma_{1}^{i} \Gamma-\gamma_{2}^{i}\right), \quad \Psi_{i}^{-}=K_{2}\left(\gamma_{2}^{i} \Gamma-\gamma_{1}^{i}\right), \quad \zeta_{i}^{+}=$ $K_{1}\left(\gamma_{1}^{i}-\gamma_{2}^{i} \Gamma\right), \zeta_{i}^{-}=K_{1}\left(\gamma_{2}^{i}-\gamma_{1}^{i} \Gamma\right), \chi_{i}^{+}=\gamma_{1}^{i} G_{1}-\gamma_{2}^{i} G_{2}-$ $\gamma_{3}^{i-1} F_{s}$, and $\chi_{i}^{-}=\gamma_{2}^{i} G_{1}-\gamma_{1}^{i} G_{2}+\gamma_{4}^{i-1} F_{s}$.

From Eq. (C1), we obtain

$$
\begin{aligned}
\frac{d^{2} M_{i}}{d \tau^{2}}= & k^{2} M_{i}+\eta_{1 i}^{+} e^{-\left(k+a_{i}\right) \tau}+\eta_{2 i}^{+} e^{\left(k-a_{i}\right) \tau}+\eta_{3 i}^{+} e^{-\left(a_{i}+1 / \mu_{0}\right) \tau} \\
& +\eta_{4 i}^{+} e^{-k \tau}+\eta_{5 i}^{+} e^{k \tau}+\eta_{6 i}^{+} e^{-\tau / \mu_{0}},
\end{aligned}
$$

$$
\begin{aligned}
\frac{d^{2} N_{i}}{d \tau^{2}}= & k^{2} N_{i}+\eta_{1 i}^{-} e^{-\left(k+a_{i}\right) \tau}+\eta_{2 i}^{-} e^{\left(k-a_{i}\right) \tau}+\eta_{3 i}^{-} e^{-\left(a_{i}+1 / \mu_{0}\right) \tau} \\
& +\eta_{4 i}^{-} e^{-k \tau}+\eta_{5 i}^{-} e^{k \tau}+\eta_{6 i}^{-} e^{-\tau / \mu_{0}}, \quad \text { (C2b) }
\end{aligned}
$$

where $\eta_{1 i}^{ \pm}=\left(\gamma_{1}^{0} \pm \gamma_{2}^{0}\right)\left(\Psi_{i}^{+} \mp \Psi_{i}^{-}\right)-\left(k+a_{i}\right)\left(\Psi_{i}^{+} \pm \Psi_{i}^{-}\right)$, $\eta_{2 i}^{ \pm}=\left(k-a_{i}\right)\left(\zeta_{i}^{+} \pm \zeta_{i}^{-}\right)+\left(\gamma_{1}^{0} \pm \gamma_{2}^{0}\right)\left(\zeta_{i}^{+} \mp \zeta_{i}^{-}\right), \eta_{3 i}^{ \pm}=\left(\chi_{i}^{+} \mp\right.$ $\left.\chi_{i}^{-}\right)\left(\gamma_{1}^{0} \pm \gamma_{2}^{0}\right)-\left(a_{i}+1 / \mu_{0}\right)\left(\chi_{i}^{+} \pm \chi_{i}^{-}\right), \eta_{4 i}^{ \pm}=-e^{-a_{i} \tau_{0} / 2}\left[\left(\gamma_{1}^{0} \pm\right.\right.$ $\left.\left.\gamma_{2}^{0}\right)\left(\Psi_{i}^{+} \mp \Psi_{i}^{-}\right)-k\left(\Psi_{i}^{+} \pm \Psi_{i}^{-}\right)\right], \eta_{5 i}^{ \pm}=-e^{-a_{i} \tau_{0} / 2}\left[k\left(\zeta_{i}^{+} \pm \zeta_{i}^{-}\right)+\right.$ $\left.\left(\gamma_{1}^{0} \pm \gamma_{2}^{0}\right)\left(\zeta_{i}^{+} \mp \zeta_{i}^{-}\right)\right]$, and $\eta_{6 i}^{ \pm}=-e^{-a_{i} \tau_{0} / 2}\left[\left(\chi_{i}^{+} \mp \chi_{i}^{-}\right)\left(\gamma_{1}^{0} \pm\right.\right.$ $\left.\left.\gamma_{2}^{0}\right)-\left(1 / \mu_{0}\right)\left(\chi_{i}^{+} \pm \chi_{i}^{-}\right)\right]$.

\section{APPENDIX D}

\section{Coefficients of Eq. (9)}

The terms $D_{1 i}, D_{2 i}, \varphi_{1 i}, \varphi_{2 i}, \varphi_{3 i}, \varphi_{4 i}, \varphi_{5 i}$, and $\varphi_{6 i}$ in Eq. (9) are $D_{1 i}^{ \pm}=A_{i} \alpha^{\overline{ }} \pm X_{i}, D_{2 i}^{ \pm}=B_{i} \alpha^{ \pm} \pm Y_{i}, \alpha^{ \pm}=(1 / 2)[1 \pm$ $\left.k /\left(\gamma_{1}^{0}+\gamma_{2}^{0}\right)\right], \quad X_{i}=\left[e^{-a_{i} \tau_{0} / 2}\left(\Psi_{i}^{+}+\Psi_{i}^{-}\right)\right] /\left[2\left(\gamma_{1}^{0}+\gamma_{2}^{0}\right)\right]-$ $\eta_{4 i}^{+} /\left[4 k\left(\gamma_{1}^{0}+\gamma_{2}^{0}\right)\right], \quad Y_{i}=\left[e^{-a_{i} \tau_{0} / 2}\left(\zeta_{i}^{+}+\zeta_{i}^{-}\right)\right] /\left[2\left(\gamma_{1}^{0}+\gamma_{2}^{0}\right)\right]+$ $\eta_{5 i}^{+} /\left[4 k\left(\gamma_{1}^{0}+\gamma_{2}^{0}\right)\right], \quad \varphi_{1 i}^{ \pm}=(1 / 2)\left(P_{i}^{+} \pm P_{i}^{-}\right), \quad \varphi_{2 i}^{ \pm}=(1 / 2)$ $\left(Q_{i}^{+} \pm Q_{i}^{-}\right), \varphi_{3 i}^{ \pm}=(1 / 2)\left(R_{i}^{+} \pm R_{i}^{-}\right), \varphi_{4 i}^{ \pm}=-\left(\eta_{4 i}^{+} \pm \eta_{4 i}^{-}\right) / 4 k$, $\varphi_{5 i}^{ \pm}=\left(\eta_{5 i}^{+} \pm \eta_{5 i}^{-}\right) / 4 k, \quad \varphi_{6 i}^{ \pm}=\left[\left(\eta_{6 i}^{+} \pm \eta_{6 i}^{-}\right) \mu_{0}^{2}\right] /\left(1-\mu_{0}^{2} k^{2}\right)$, $P_{i}^{ \pm}=\eta_{1 i}^{ \pm} /\left[\left(k+a_{i}\right)^{2}-k^{2}\right], Q_{i}^{ \pm}=\eta_{2 i}^{ \pm} /\left[\left(k-a_{i}\right)^{2}-k^{2}\right]$, and $R_{i}^{ \pm}=\eta_{3 i}^{ \pm} /\left[\left(a_{i}+1 / \mu_{0}\right)^{2}-k^{2}\right]$. We determine $B_{i}$ and $A_{i}$ by the boundary conditions as 


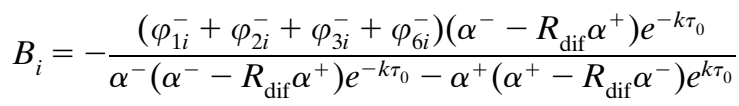

$$
\begin{aligned}
& +\frac{\alpha^{+}\left[\left(\varphi_{1 i}^{+}-R_{\mathrm{dif}} \varphi_{1 i}^{-}\right) e^{-\left(k+a_{i}\right) \tau_{0}}+\left(\varphi_{2 i}^{+}-R_{\mathrm{dif}} \varphi_{2 i}^{-}\right) e^{\left(k-a_{i}\right) \tau_{0}}+\left(\varphi_{3 i}^{+}-R_{\mathrm{dif}} \varphi_{3 i}^{-}\right) e^{-\left(a_{i}+1 / \mu_{0}\right) \tau_{0}}\right]}{\alpha^{-}\left(\alpha^{-}-R_{\mathrm{dif}} \alpha^{+}\right) e^{-k \tau_{0}}-\alpha^{+}\left(\alpha^{+}-R_{\mathrm{dif}} \alpha^{-}\right) e^{k \tau_{0}}}
\end{aligned}
$$

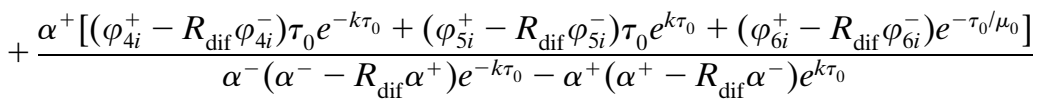

$$
\begin{aligned}
& +\frac{\left(X_{i}+Y_{i}\right)\left(\alpha^{-}-R_{\mathrm{dif}} \alpha^{+}\right) e^{-k \tau_{0}}+\left(X_{i}+R_{\mathrm{dif}} X_{i}\right) \alpha^{+} e^{-k \tau_{0}}+\left(Y_{i}+R_{\mathrm{dif}} Y_{i}\right) \alpha^{+} e^{k \tau_{0}}}{\alpha^{-}\left(\alpha^{-}-R_{\mathrm{dif}} \alpha^{+}\right) e^{-k \tau_{0}}-\alpha^{+}\left(\alpha^{+}-R_{\mathrm{dif}} \alpha^{-}\right) e^{k \tau_{0}}},
\end{aligned}
$$

$$
A_{i}=\frac{1}{\alpha^{+}}\left(X_{i}+Y_{i}-B_{i} \alpha^{-}-\varphi_{1 i}^{-}-\varphi_{2 i}^{-}-\varphi_{3 i}^{-}-\varphi_{6 i}^{-}\right)
$$

\section{REFERENCES}

Arai, T., 1965: On the relationship between albedo and the properties of snow cover. Research Institute for Natural Resources Miscellaneous Rep. 64, 12-19.

Boutle, I. A., S. J. Abel, P. G. Hill, and C. J. Morcrette, 2014: Spatial variability of liquid cloud and rain: Observations and microphysical effects. Quart. J. Roy. Meteor. Soc., 140, 583594, https://doi.org/10.1002/qj.2140.

Chen, R., R. Wood, Z. Li, R. Ferraro, and F.-L. Chang, 2008: Studying the vertical variation of cloud droplet effective radius using ship and space-borne remote sensing data. J. Geophys. Res., 113, D00A02, https://doi.org/10.1029/ 2007JD009596.

Cheng, M. N., C. S. Lu, and Y. G. Liu, 2015: Variation in entrainment rate and relationship with cloud microphysical properties on the scale of $5 \mathrm{~m}$. Sci. Bull., 60, 707-717, https:// doi.org/10.1007/s11434-015-0737-8.

Colbeck, S. C., 1975: Grain and bond growth in wet snow. Snow Mechanics: Proceedings of the Grindelwald Symposium, IAHS Publ. 114, 51-61.

de Haan, J. F., P. B. Bosma, and J. W. Hovenier, 1987: The adding method for multiple scattering calculations of polarized light. Astron. Astrophys., 183, 371-391.

Flanner, M. G., and C. S. Zender, 2006: Linking snowpack microphysics and albedo evolution. J. Geophys. Res., 111, D12208, https://doi.org/10.1029/2005JD006834.

Fu, Q., K. N. Liou, M. C. Cribb, T. P. Charlock, and A. Grossman, 1997: Multiple scattering parameterization in thermal infrared radiative transfer. J. Atmos. Sci., 54, 2799-2812, https://doi.org/ 10.1175/1520-0469(1997)054<2799:MSPITI>2.0.CO;2.

Gow, A. J., 1969: On the rates of growth of grains and crystals in South Polar firn. J. Glaciol., 8, 241-252, https://doi.org/ 10.3189/S0022143000031233.

Guo, J. P., and Coauthors, 2016: Three-dimensional structure of aerosol in China: A perspective from multi-satellite observations. Atmos. Res., 178-179, 580-589, https://doi.org/10.1016/ j.atmosres.2016.05.010.

Hansen, J. E., 1971: Multiple scattering of polarized light in planetary atmospheres. Part I. The doubling method. J. Atmos. Sci., 28, 120-125, https://doi.org/10.1175/1520-0469(1971)028<0120: MSOPLI $>2.0 . C O ; 2$.
Hobbs, P. V., 1974: Ice Physics. Clarendon Press, 837 pp.

Kato, T., 1966: Perturbation Theory for Linear Operators. Springer-Verlag, $592 \mathrm{pp}$.

LaChapelle, E. R., 1969: Field guide to snow crystals. Arct. Alp. Res., 207, 464-465.

Lenoble, J., 1985: Radiative Transfer in Scattering and Absorbing Atmospheres: Standard Computational Procedures. A. Deepak Publishing, $314 \mathrm{pp}$.

Letu, H., T. Y. Nakajima, and T. N. Matsui, 2012: Development of an ice crystal scattering database for the Global Change Observation Mission/Second Generation Global Imager Satellite Mission: Investigating the refractive index grid system and potential retrieval error. Appl. Opt., 51, 6172-6178, https://doi.org/ 10.1364/AO.51.006172.

_ , H. Ishimoto, J. Riedi, T. Y. Nakajima, L. C. Labonnote, A. Baran, T. M. Nagao, and M. Sekiguchi, 2016: Investigation of ice particle habits to be used for ice cloud remote sensing for the GCOM-C satellite mission. Atmos. Chem. Phys., 16, 12 287-12 303, https://doi.org/10.5194/acp-16-12287-2016.

Li, J., D. J. W. Geldart, and P. Chýlek, 1994: Solar radiative transfer in clouds with vertical internal homogeneity. J. Atmos. Sci., 51, 2542-2552, https://doi.org/10.1175/1520-0469(1994)051<2542: SRTICW $>2.0 . \mathrm{CO} ; 2$.

—, S. Dobbie, P. Räisänen, and Q. Min, 2005: Accounting for unresolved clouds in a 1-D solar radiative-transfer model. Quart. J. Roy. Meteor. Soc., 131, 1607-1629, https://doi.org/ 10.1256/qj.04.31.

_ - X. Ma, K. von Salzen, and S. Dobbie, 2008: Parameterization of sea-salt optical properties and physics of the associated radiative forcing. Atmos. Chem. Phys., 8, 4787-4798, https:// doi.org/10.5194/acp-8-4787-2008.

Liou, K. N., 2002: An Introduction to Atmospheric Radiation. 3rd ed. Academic Press, 583 pp.

Luo, Z. J., J. Jeyaratnam, S. Iwasaki, H. Takahashi, and R. Anderson, 2014: Convective vertical velocity and cloud internal vertical structure: An A-Train perspective. Geophys. Res. Lett., 41, 723-729, https://doi.org/10.1002/2013GL058922.

Macke, A., D. L. Mitchell, and L. V. Bremen, 1998: Monte Carlo radiative transfer calculations for inhomogeneous mixed phase clouds. Phys. Chem. Earth, 24B, 237-241, https://doi.org/ 10.1016/S1464-1909(98)00044-6.

Meador, W. E., and W. R. Weaver, 1980: Two-stream approximations to radiative transfer in planetary atmospheres: A unified description of existing methods and a new improvement. J. Atmos. Sci., 37, 630-643, https://doi.org/10.1175/ 1520-0469(1980)037<0630:TSATRT>2.0.CO;2.

Mellor, M., 1977: Engineering properties of snow. J. Glaciol., 19, 15-66, https://doi.org/10.3189/S002214300002921X. 
Nakajima, T., M. Tsukamoto, Y. Tsushima, A. Numaguti, and T. Kimura, 2000: Modeling of the radiative process in an atmospheric general circulation model. Appl. Opt., 39, 48694878, https://doi.org/10.1364/AO.39.004869.

Nakaya, U., 1954: Snow Crystals: Natural and Artificial. Harvard University Press, 510 pp.

Namazi, N., K. von Salzen, and J. N. S. Cole, 2015: Simulation of black carbon in snow and its climate impact in the Canadian Global Climate Model. Atmos. Chem. Phys., 15, 1088710 904, https://doi.org/10.5194/acp-15-10887-2015.

Noonkester, V. R., 1984: Droplet spectra observed in marine stratus cloud layers. J. Atmos. Sci., 41, 829-845, https://doi.org/ 10.1175/1520-0469(1984)041<0829:DSOIMS > 2.0.CO;2.

Qin, K., and Coauthors, 2016: Trans-boundary aerosol transport during a winter haze episode in China revealed by groundbased lidar and CALIPSO satellite. Atmos. Environ., 141, 20-29, https://doi.org/10.1016/j.atmosenv.2016.06.042.

_- L. Wang, L. Wu, J. Xu, L. Rao, H. Letu, T. Shi, and R. Wang, 2017: A campaign for investigating aerosol optical properties during winter hazes over Shijiazhuang, China. Atmos. Res., 198, 113-122, https://doi.org/10.1016/j.atmosres.2017.08.018.

Sommerfeld, R. A., and E. LaChapelle, 1970: The classification of snow metamorphism. J. Glaciol., 9, 3-18, https://doi.org/ 10.3189/S0022143000026757.

Toon, O. B., C. P. McKay, T. P. Ackerman, and K. Santhanam, 1989: Rapid calculation of radiative heating rates and photodissociation rates in inhomogeneous multiple scattering atmospheres. J. Geophys. Res., 94, 16287-16301, https://doi.org/ 10.1029/JD094iD13p16287.

Vane, D., N. Tourville, G. Stephens, and A. Kankiewicz, 2006: New observations of hurricanes from the CloudSat radar. Eos, Trans. Amer. Geophys. Union, 87 (Fall Meeting Suppl.), Abstract A13A-0885.

von Salzen, K., and Coauthors, 2013: The Canadian Fourth Generation Atmospheric Global Climate Model (CanAM4).
Part I: Representation of physical processes. Atmos.-Ocean, 51, 104-125, https://doi.org/10.1080/07055900.2012.755610.

Wang, F., and Coauthors, 2015: Multi-sensor quantification of aerosol-induced variability in warm cloud properties over eastern China. Atmos. Environ., 113, 1-9, https://doi.org/ 10.1016/j.atmosenv.2015.04.063.

Warren, S. G., and W. J. Wiscombe, 1980: A model for the spectral albedo of snow. II: Snow containing atmospheric aerosols. J. Atmos. Sci., 37, 2734-2745, https://doi.org/ 10.1175/1520-0469(1980)037<2734:AMFTSA > 2.0.CO;2.

Wiscombe, W. J., and S. G. Warren, 1980: A model for the spectral albedo of snow. I: Pure snow. J. Atmos. Sci., 37, 2712-2733, https://doi.org/10.1175/1520-0469(1980)037<2712: AMFTSA $>2.0 . \mathrm{CO} ; 2$.

Yang, P., K. Liou, L. Bi, C. Liu, B. Yi, and B. Baum, 2015: On the radiative properties of ice clouds: Light scattering, remote sensing, and radiation parameterization. Adv. Atmos. Sci., 32, 32-63, https://doi.org/10.1007/s00376-014-0011-z.

Young, A. H., J. Bates, and J. Curry, 2013: Application of cloud vertical structure from CloudSat to investigate MODISderived cloud properties of cirriform, anvil, and deep convective clouds. J. Geophys. Res. Atmos., 118, 4689-4699, https://doi.org/10.1002/jgrd.50306.

Zhang, F., and J. Li, 2013: Doubling-adding method for delta-fourstream spherical harmonic expansion approximation in radiative transfer parameterization. J. Atmos. Sci., 70, 3084-3101, https://doi.org/10.1175/JAS-D-12-0334.1.

— Z Z. Shen, J. Li, X. Zhou, and L. Ma, 2013: Analytical deltafour-stream doubling-adding method for radiative transfer parameterizations. J. Atmos. Sci., 70, 794-808, https://doi.org/ 10.1175/JAS-D-12-0122.1.

Zhang, J. L., J. Shang, and G. Y. Zhang, 2017: Verification for different contrail parameterizations based on integrated satellite observation and ECMWF reanalysis data. Adv. Meteor., 2017, 8707234, https://doi.org/10.1155/2017/8707234. 